\title{
Cultural Norms, the Phenomenology of Incorporation and the Experience of Having a Child Born with Ambiguous Sex.
}

Kristin Zeiler and Erik Malmqvist

\section{Linköping University Post Print}

N.B.: When citing this work, cite the original article.

This is an electronic version of an article published in:

Kristin Zeiler and Erik Malmqvist, Cultural Norms, the Phenomenology of Incorporation and the Experience of Having a Child Born with Ambiguous Sex., 2010, IUC Journal of Social Work Theory and Practice, (36), 1, 157-164.

IUC Journal of Social Work Theory and Practice is available online at informaworldTM:

http://dx.doi.org/

Copyright: Taylor \& Francis

http://www.tandf.co.uk/journals/default.asp

Postprint available at: Linköping University Electronic Press

http://urn.kb.se/resolve?urn=urn:nbn:se:liu:diva-64038 


\section{Cultural Norms, the Phenomenology of Incorporation and the Experience of Having a}

\section{Child Born with Ambiguous Sex}

\section{Malmqvist and Zeiler in Social Theory and Practice 2010.}

One of the first questions likely to arise when a child is born is whether it is a boy or a girl. This is not always quite as easily determined as one might think. Due to one of a number of different conditions a baby may have genitals which do not have an unambiguously male or female appearance, or which appear to belong to one sex while chromosomes or hormone levels belong to the opposite sex. In such cases, parents are soon after delivery asked to decide, together with medical staff, about the child's gender identity and also about surgical intervention. Should the child be defined as a boy or a girl? And should the child undergo surgery so that its genitals become more typical of the assigned sex? Recent empirical research shows that parents experience this situation as profoundly confusing and frustrating. ${ }^{1}$ They are typically greatly relieved when the child is assigned a sex. Also, they often consent to genital surgery for their child, despite the fact that such surgery is in most cases not medically necessary and despite the risk that it may impair the child's future sexual sensation or function.

One plausible way to make sense of the parents' experiences and choices in this kind of case is with reference to pervasive cultural norms about sexual difference and the importance of genital appearance for someone's belonging to either sex. The parents feel what

\footnotetext{
${ }^{1}$ Brendan Gough, Nicky Weyman, Julie Alderson, Gary Butler \& Mandy Stoner, “They did not have a word': The parental quest to locate a 'true sex' for their intersex children," Psychology and Health 23(4) (2008): 493-507; and Caroline Sanders, Bernie Carter \& Lynne Goodacre, 'Parents' narratives about their experiences of their child's reconstructive genital surgeries for ambiguous genitalia," Journal of Clinical Nursing 17(23) (2008): 3187-95.
} 
they feel and do what they do partly or largely because of the influence of such norms. ${ }^{2}$ This account raises a set of difficult, interrelated questions. How, more precisely, do cultural norms influence the parents' experiences and actions? And just how deep is that influence? Could the parents resist it? If the child is harmed by the genital surgery, are they morally responsible? More generally, the parents' response to an ambiguously sexed child illustrates a basic but thorny problem that haunts philosophy and social theory alike: how is the relationship between individual agency and cultural patterns of understanding and behaviour to be understood? This is not just a challenging academic issue, but also a pressing problem for feminists and other social critics concerned with challenging and changing such patterns.

In this paper, we examine the interplay between pervasive cultural norms and individual actions. The paper is divided in two main parts, the first more theoretical, the second more applied. In sections 3 and 4 we elaborate an account of how norms sometimes shape people's experiences and actions. Then, in section 5, we draw on this account in order to discuss in more detail the parental experiences of and responses to the birth of a child with ambiguous sex briefly described above. But first of all we outline the theoretical background to our discussion (section 1) and our own theoretical point of departure (section 2).

\section{Cultural Norms and Individual Agency in the Autonomy Debate}

\footnotetext{
${ }^{2}$ See e.g. Ellen K. Feder, “'In Their Best Interests’: Parents’ Experience of Atypical Genitalia,” in Erik Parens (ed.), Surgically Shaping Children: Technology, Ethics, and the Pursuit of Normality (Baltimore: The Johns Hopkins University Press, 2006), pp. 189-210. As Feder points out, the influence of these norms on medical practice and medical personnel may be at least as determinative for what happens in this situation as their influence on the parents.
} 
The problem of how to think about the relationship between cultural norms and individual actions has been posed and grappled with within different theoretical frameworks. ${ }^{3}$ One context where the problem, and feminists' concern with it, are particularly emphasised is the contemporary philosophical debate about personal autonomy. In that debate, autonomy is often understood in procedural terms - as consisting in, or at least as involving as a central feature, a capacity to critically examine and revise one's motivational structure. From this perspective, preferences do not have to have particular content in order to count as autonomous. Procedural autonomy is thus not to be confused with ideals of individual self-sufficiency, as one may well autonomously choose to depend on others. Nor is it sufficient for autonomy simply to do what one wants. What does matter is that whatever preferences that one acts on are adequately reflected upon and accepted as preferences that one really wants, or can identify with, in the light of more important values and commitments. ${ }^{4}$

${ }^{3}$ One theoretical framework that requires mentioning in particular is that which the French social theorist Pierre Bourdieu develops in a range of works. See e.g. Pierre Bourdieu, Outline of a Theory of Practice, trans. Richard Nice (Cambridge: Cambridge University Press, 1977). Bourdieu's thought has been applied to parents' experiences of children with ambiguous sex; see Feder, "In Their Best Interests". There are close affinities between this theoretical perspective and the one that we rely on. MerleauPonty's phenomenology has had an important impact on Bourdieu's sociology, and it can be used to strengthen it at crucial points; see Nick Crossley, "The phenomenological habitus and its construction," Theory and Society 30 (2001): 81-120. A different approach to norms and normalisation (perhaps less concerned than we are with individual agency and more with discursive and institutional dimensions of human life) that might require mentioning is the Foucauldian one. Notably, Foucault himself has discussed ambiguous sex; see Michel Foucault, "Introduction," in Herculine Barbin: Being the Recently Discovered Memoir of a Nineteenth Century French Hermaphrodite, trans. Richard McDougall (New York: Pantheon, 1980), pp. vii-xvii.

${ }^{4}$ For two well-known accounts of autonomy along these lines, see Gerald Dworkin, The Theory and Practice of Autonomy (Cambridge: Cambridge University Press, 1988); and Harry Frankfurt, "Freedom of the 
Feminists have argued that this model does not adequately account for how autonomy is hampered by oppressive socialisation and pervasive gender norms. It runs up against what Natalie Stoljar calls "the feminist intuition", i.e. the idea that preferences that are influenced by oppressive norms of femininity cannot be considered autonomous. ${ }^{5}$ She argues that women who choose not to use contraceptives because they are motivated by internalised and questionable norms about female sexuality and the importance of motherhood for women's worth are not acting autonomously, even if they reflect thoroughly on their motives and accept them as their own. This is because they do not recognise the morally objectionable content of these norms. Paul Benson has presented an analogous argument with respect to women's compliance to norms concerning female physical appearance. ${ }^{6}$

This underscores that, from a feminist point of view, it is on the one hand important not to think of the impact of pervasive cultural norms in overly voluntaristic terms. An unqualified procedural model invites us to think of deeply entrenched norms as influencing our actions by way of preferences that are as open to voluntary critical scrutiny and revision as any other motives. But this appears to miss the feminist point that when we comply with such norms our motives are sometimes more intractable or less transparent than other motives. On the other hand, however, one should be careful not to go too far towards the opposite extreme either: it

Will and the Concept of a Person," Journal of Philosophy 68(1) (1971): 5-20. (Note, however, that Frankfurt speaks of free will rather than autonomy.) Many critics of such accounts agree that autonomy involves a necessary procedural element, but argue that this element is not sufficient. See, for instance, Paul Benson, “Autonomy and Oppressive Socialization," Social Theory and Practice 17(3) (1991): 385-408; and Natalie Stoljar “Autonomy and the Feminist Intuition”, in Catriona Mackenzie and Natalie Stoljar (eds.), Relational Autonomy: Feminist Perspectives on Autonomy, Agency and the Social Self (New York: Oxford University Press, 2000), pp. 94-111.

\footnotetext{
${ }^{5}$ Stoljar, "Autonomy and the Feminist Intuition".

${ }^{6}$ Benson, “Autonomy and Oppressive Socialization”.
} 
would be a mistake to think of the relationship between cultural norms and actions in causal or quasi-causal terms. Most feminists agree that although norms may constrain our choices, they do not strictly speaking force us to act in any particular way. Indeed, this insight is crucial to the whole feminist project. If oppressive values, norms and practices simply determined our behaviour through some causal mechanism there would be little point in trying to resist them, or in trying to convince other people to resist them, on particular occasions. And that is part of what feminism is about.

In the following sections, we will elaborate an alternative to the two just outlined ways in which one might think about how norms influence people's actions - an alternative that escapes the voluntarism of the first line of thought without subscribing to the determinism of the second. We intend to explain, that is, both how pervasive cultural norms may elude autonomous reflection and issue in non-autonomous agency in ways that other sources of motivation do not, and how we may nevertheless sometimes relate critically to such norms and avoid their shaping our actions. Our approach is phenomenological, and takes its point of departure in the French philosopher Maurice Merleau-Ponty's phenomenology of the body, as he develops it in his celebrated Phenomenology of Perception (first published in 1945 with the title Phénoménologie de la perception).

\section{Merleau-Ponty's Phenomenology of the Body}

Phenomenology of Perception constitutes an ambitious take on a range of long-standing and intricate philosophical problems, such as the nature of perceptual experience, the character of human behaviour and the existence of other selves and an outside world, as well as a trenchant critique of many of the ways in which these problems have been treated in Western thought. At the heart of the argument, reappearing at each of its junctures, stands his phenomenological analysis of the irreducibly bodily nature of human being-in-the-world. For Merleau-Ponty, it is only because we inhabit a body that pre-reflectively opens up a world of meaning to us that we can approach 
things and people around us in perception, action, language and thought. Our body is our "anchorage in a world," 7 that by which it is possible for us to have a world at all.

This view is in stark contrast with the Cartesian conception of mind and body as radically metaphysically distinct, the one conscious and non-material, the other material and nonconscious. It is also notably at odds with conceptions of the body in many more recent philosophical and scientific schools of thought. The body that Merleau-Ponty describes is not a passive receiver of sensory input, as it is characterised by empiricist analyses of perception, or merely the locus of mechanical reflexes to physical stimuli, as behaviourists have argued. In short, it is not a mere piece of matter, placed alongside other pieces of matter in a network of causal relations. Instead, it is the body as we live it in first-person experience. Its relation to the things in the world and to other people is not primarily one of cause and effect, but one of meaning or intention. As bodily creatures we are always situated in circumstances that we are capable of actively responding to and transcending.

So according to Merleau-Ponty, my body does not exist as an object for me, and the world in which I am corporeally involved is not the objective world of the natural sciences. On the contrary, I am my body, ${ }^{8}$ and as a body I act upon and perceive the world as a system of possibilities. It is important to note that this involves as radical a departure from transcendental or idealist philosophies as it does from the various forms of objectivist thought already mentioned. If we are bodily subjects, then subjectivity cannot be understood in terms of a pure and transparent consciousness, a transcendental "for itself", set apart from and constituting its world as an "in itself". We are not primarily knowledge-subjects, and the world around us is not primarily a thought-about or represented world. For Merleau-Ponty, our basic mode of being in the world is instead practical and pre-reflective. He writes: "Consciousness is not in the first place a

\footnotetext{
${ }^{7}$ Maurice Merleau-Ponty, Phenomenology of Perception, trans. Colin Smith (London: Routledge, 2002), p. 167.

8 Ibid., p. 231.
} 
matter of 'I think that' but of 'I can'."' Of course, we can approach things in explicit and thematic thought too, but such an approach presupposes an immediate and pre-thematic bodily familiarity with the world as its condition of possibility.

Merleau-Ponty's emphasis on the primordial practical and pre-reflective layer of human existence is particularly well articulated in his reconceptualisation of the psychological notion of the body schema. He uses the term to describe how in movement the body seamlessly but tacitly coordinates its different parts, and at the same time draws up a space into which it projects itself. That is, the body schema, as Merleau-Ponty understands it, is an implicit awareness which is rooted in motility, and by virtue of which the lived body simultaneously forms a whole and aligns itself with its surroundings. He writes: "it is not only an experience of my body, but an experience of my body-in-the-world."10

As an illustration, suppose that I reach for a glass of water standing on my desk. Through a carefully adjusted flexion of my elbow and shoulder, my upper and lower arm traverse just the right distance in just the right direction to put the glass within reach of my hand, and my fingers stretch and grip in ways minutely geared towards seizing hold of the glass, while the muscles of my back and chest support the whole movement. And correspondingly, the glass itself stands out from the background made up of my desk and the other things that occupy it, as the end-point of my movement, while the distance that separates it from my hand shrinks into a distance-to-be-crossed. All of this may well take place without my explicit attention. Nonetheless, there is certainly a sort of knowledge or awareness involved. My movement is intelligible and infused with intentionality, and any of its constitutive moments may become the object of conscious scrutiny and control. This may happen, for instance, if my fingers slip when trying to seize the glass, threatening to tip it over and pour water all over my desk.

\footnotetext{
${ }^{9}$ Ibid., p. 159.

${ }^{10}$ Ibid., pp. 163-4.
} 
Of what use might Merleau-Ponty's theory of the lived body be for coming to grips with the problem of the influence of pervasive cultural norms on our actions that we described in the previous section? Quite generally, it appears to offer an attractive middle way between the two unsatisfactory ways of thinking of such influence outlined there. If cultural norms operate on the largely pre-reflective, but nonetheless intentional and meaningful level of embodied being-inthe-world, we need not think of their influence on actions either in terms of explicitly thoughtabout motives or in terms of some unintelligible and rigid causal mechanism. We can, it would seem, recognise their hold on us as strong and elusive, while also leaving open the possibility that we sometimes can reflect on and choose to resist them, i.e. exercise autonomy with respect to them.

A connection along these lines between social and cultural patterns and individual actions is indeed hinted at by Merleau-Ponty himself in his insistence that the world in which we are corporeally situated, and which we respond to and transcend in meaningful ways, is always already a cultural and inter-subjective world. ${ }^{11}$ And other authors have described more explicitly how cultural patterns are played out in the lived body. For instance, Iris Marion Young argues that patriarchal society's tendency to objectify women has contributed to shaping a distinct feminine bodily existence that is inhibited in various ways - that is marked, for instance, by a tentative and self-conscious mode of transcendence to the surrounding world. ${ }^{12}$ And Charles Taylor suggests that social life relies on a form of understanding that is inarticulate and embodied (rather than explicit and representational), and that this is crucial for the social sciences if they are

\footnotetext{
${ }^{11}$ See e.g. ibid., pp. 405-15.

12 Iris Marion Young, "Throwing Like a Girl: A Phenomenology of Feminine Bodily Comportment, Motility and Spatiality," Human Studies 3(1) (1980): 137-56.
} 
to make sense of regularities in human behaviour, of what goes on, for instance, when people follow rules or accede to norms. ${ }^{13}$

The idea that cultural patterns make their way into the lived body, and that the body is our medium for our making our way in cultural space, is important. But this idea needs to be fleshed out considerably if it is to be of any help in solving the problem that this paper is concerned with. How, more precisely, are pervasive cultural norms taken up and lived as part of our bodily existence? What difficulties do they pose for autonomous reflection and agency? And how can we nonetheless relate critically to them - i.e. what possibilities are there of spotting them and preventing them from shaping our actions? The approach to these questions will begin by a consideration of what Merleau-Ponty and other phenomenologists have to say about incorporation.

Before turning to the details of the analysis, however, we need to make an important qualification. The phenomenology of the body is more apt for elucidating the influence of some cultural norms than the influence of others. ${ }^{14}$ The culturally shared practices that our analysis is meant to capture all partly and primarily require various kinds of bodily

${ }^{13}$ Charles Taylor, “To Follow a Rule...," in Craig J. Calhoun, Edward LiPuma \& Moishe Postone (eds.), Bourdieu: Critical Perspectives (Cambridge: Polity Press, 1993). Taylor's ambition in the cited paper closely resembles our own: he wants to show how thinking about human social behaviour in terms of embodied understanding is an alternative both to thinking about it as causally determined and to conceiving of it in terms of explicit, representational thought. The reason why we do not follow him further than we do is that he, in that paper, says little about how such understanding is appropriated, and about how it may both elude and lend itself to explicit articulation. These are crucial issues if we are to understand how we may relate, and fail to relate, critically or autonomously to it.

${ }^{14}$ We thank one of this journal's anonymous reviewers for convincing us to make this important qualification and for suggesting the property right norm as a possible counterexample to our analysis. 
movement, comportment or expression. This is by no means a small category: most norms that govern everyday, face-to-face social interaction arguably involve important such elements. This is certainly true of many of the deeply rooted norms that feminist scholars have discussed and criticised. For instance, in order to live up to society's standards of female physical appearance, women are required to engage in, as Benson puts it, "prolonged efforts at plucking, painting, toning, tanning, starving and meticulously scrutinizing their bodies"15.

Other norms primarily hold sway on more institutional and discursive levels of social life. Consider, for instance, the property right norm that has a prominent place in American society. It seems that this norm too is sometimes expressed in individual embodiment - think of the landowner's physical agitation at the sight of a trespasser - but its most important influence is elsewhere, for instance on legislation and political discourse. An analysis of how such norms are corporeally manifested may not be altogether misplaced ${ }^{16}$, but it does not capture what is most important about them. Consequently, it is not primarily the influence of norms of this kind that we are concerned with.

\section{Incorporation, Habit and Cultural Norms}

The body schema, as Merleau-Ponty describes it, is a changeable structure. He discusses at great length how our sensori-motor capacities may be diminished or inhibited as a result of illness or injury, and how the world that they open up to us is then correspondingly impoverished and constrained in various ways. But the body schema can also be transformed in a more positive manner, as the following example illustrates:

\footnotetext{
${ }^{15}$ Benson, “Autonomy and Oppressive Socialization”, p. 386.

${ }^{16}$ Merleau-Ponty insists throughout Phenomenology of Perception that even the most intellectual and discursive kinds of human activity rely on pre-reflective embodiment. If this is right, we should expect even these activities to correspond to specific corporeal styles. Although an investigation of these styles would be interesting, we will not pursue it here.
} 
The blind man's stick has ceased to be an object for him, and is no longer perceived for itself; its point has become an area of sensitivity, extending the scope and active radius of touch, and providing a parallel to sight. In the exploration of things, the length of the stick does not enter expressly as a middle term: the blind man is rather aware of it through the position of objects than of the position of objects through it. ${ }^{17}$

We might say that the blind man has incorporated the stick. It is no longer encountered as a thing out there in the world, but integrates itself with and extends the bodily resources with which he makes sense of the surrounding world and acts upon it. There are two features of this incorporation that should be noted in particular. First, when incorporated, the stick creates new perceptual and motor possibilities for the blind man. It allows him to perceive things that were wholly beyond reach before he learned to make use of it and allows him to perceive already familiar things in new ways, and the space in which he finds himself is as a result both expanded and qualitatively changed. Things are given more definite locations and more detailed structures, and he can approach them and make his way among them more confidently.

Second, the incorporation of the stick involves its receding from attention. This feature is not just accidentally related to the feature just mentioned, but is rather its necessary correlate. Suppose that the blind man is crossing an unfamiliar room with the help of his stick. Through the stick he has a sense of the location of various pieces of furniture placed in the room and of various objects scattered across the floor, and this allows him to navigate safely between them. But this requires that the stick remains a medium for his experience of the room, rather than itself coming to the fore as the focus of the experience. If suddenly the man concentrates on

\footnotetext{
${ }^{17}$ Merleau-Ponty, Phenomenology of Perception, pp. 165-66.
} 
the stick itself, the sense of the room in which he moves is disrupted, and he is required to stop or else is at risk of stumbling over or bumping into something.

This transparency of incorporated objects is simply an extension of that very same feature of the lived body into which they are incorporated. Our bodily perception of and action upon the world relies on what Drew Leder calls "focal disappearance": "the self-effacement of bodily organs when they form the focal origin of a perceptual or actional field." ${ }^{18}$ A simple example of this phenomenon is the absence of the eyes from the visual field. Another example is the withdrawal of my hands from explicit awareness when I type a letter. The typing proceeds as long as I concentrate on the words and sentences that I try to formulate, but is temporarily interrupted as soon as I instead shift attention to the hands with which I type. As Leder illuminatingly puts it (drawing on Michael Polanyi), my eyes or hands are that from which I attend to something else - the things I see or the letter that I am writing. ${ }^{19}$ I cannot simultaneously attend to the organ in question and from that organ to something else; I cannot "see my seeing". The incorporation of an external object is the absorption of that object into the focal disappearance and the "from structure" of the acting and perceiving body. ${ }^{20}$ The object becomes part of that from which we act and perceive, and as such it recedes from view.

This absorption, however, is less than total. Don Ihde points out that our relation to incorporated instruments is "semi-transparent". Even as they recede from thematic attention as we engage with the world through them, we retain a certain implicit awareness of them as our instruments - an "echo focus". ${ }^{21}$ The blind man is for instance vaguely conscious of the stick in

\footnotetext{
${ }^{18}$ Drew Leder, The Absent Body (Chicago: The University of Chicago Press, 1990), p. 26.

${ }^{19}$ Ibid., pp. 15-17.

${ }^{20}$ Ibid., p. 33.

${ }^{21}$ Don Ihde, Technics and Praxis (Dordrecht: Riedel, 1979), pp. 19-20.
} 
his hand even as he uses it to make his way through the room. But the more skilled he is in using it, the more transparent it becomes. ${ }^{22}$

Not only simple tools, such as the stick in Merleau-Ponty's example, are capable of becoming incorporated. A more complex piece of technology like a telephone is analogously a transparent medium that extends and shapes our experiential possibilities: through it the voice of a geographically distant friend is made present, although in a different way than in an ordinary face-to-face conversation. ${ }^{23}$ We shall suggest that it is sometimes useful to think of cultural norms as incorporated too. ${ }^{24}$ Remember that the norms that we are concerned with are those that are to some degree corporeally enacted. Note also that since the influence even of norms of this particular type is likely to be complex, an analysis in terms of incorporation cannot be expected to capture all aspects of that influence. But it nevertheless brings out important features of how individual agents appropriate these norms, and how their experience is shaped by them.

An analogy between incorporating material objects like sticks and telephones and complying with cultural norms may appear to imply an implausibly reified view of the latter. In

22 The degree of transparency of an instrument does not merely depend on the user's skill, but also, as Ihde points out, on the degree of perfection of the instrument itself. See Ihde, Technics and Praxis, p. 20. It should also be noted that the parts of the body with which instruments participate in focal disappearance are not themselves rendered completely transparent through such disappearance; we retain a certain "coenesthetic awareness" of them. See Leder, The Absent Body, p. 23.

${ }^{23}$ Ihde, Technics and Praxis, p. 24.

${ }^{24}$ The step from sticks and telephones to cultural norms may at first blush appear overly bold, but we hope to make clear in the following discussion that it is not. Other authors, too, have extended the analysis of incorporation far beyond technological examples, but in other directions than ours. Crossley, for instance, suggests that certain social relationships can be understood in such terms: groups with which we identify may for instance be swallowed up in our taken-for-granted bodily existence, and become part of the point of view from which we make sense of the world. See Crossley, “The phenomenological habitus and its construction," pp. 103-104. 
order to see why this is not the case we need to consider an important aspect of incorporation left out in the above characterisation: its intimate connection to babit. Indeed, when MerleauPonty introduces the example of the blind man's stick it is primarily the concept of habit that he seeks to explicate. Acquiring a skill or developing a habit involves just the kind of transformation of the corporeal schema that we have seen that the bodily appropriation of objects brings about. In the process of learning how to dance, ${ }^{25} \mathrm{I}$ gradually develop new living intra-corporeal and body-world relationships. I acquire the capacity to coordinate my arms, hips and feet in new ways. And this parallels a new sense of coordination with my surroundings: my body picks up the rhythm of the music, and I can move about the dance-floor without stepping on my partner's feet.

Properly understood, incorporation and habituation are not so much two similar but isolated phenomena, but rather two closely intertwined elements of the same process. The transformation of the corporeal schema involved in acquiring a skill like dancing implies the appropriation of something initially external to it - a certain practical logic manifested in the movements of a more competent dancing instructor or partner, or at least hinted at by the music one hears. ${ }^{26}$ And conversely, even the incorporation of simple objects requires certain practical familiarisation with them. Before the blind man can make the stick the medium for his experience - before he can attend to the world through the stick - he needs to grow accustomed to its feel in

25 This is another of Merleau-Ponty's examples. See Merleau-Ponty, Phenomenology of Perception, p. 165.

26 This way of putting it closely resembles Crossley's explication of the Merleau-Pontean notion of habit. See Crossley, "The phenomenological habitus and its construction”, pp. 104-107. Merleau-Ponty himself describes the acquisition of skills and habits as incorporation: "It is literally true that the subject who learns to type incorporates the key-bank space into his bodily space". See Phenomenology of Perception, p. 167. See also Leder, The Absent Body, pp. 30-32. 
his hand and to the novel ways in which it reveals to him the structure and position of objects around him. ${ }^{27}$

So habituation can be understood as the temporal dimension of incorporation - the gradual sedimentation of past activity, whereby that activity simultaneously withdraws itself into the flesh and unlocks new experiential possibilities. ${ }^{28}$ As I progress as a dancer I need to focus less and less on my own steps or those of my instructor, and I am increasingly able to direct my attention elsewhere: to the music being played, to a conversation with my partner, and so on. My movements are gradually taken up into the taken-for-granted bodily resources with which I engage the world. I come to view things partly from the perspective of an amateur dancer - the dance floor, for instance, takes on a more inviting and less intimidating character. As MerleauPonty points out, in keeping with his critique of both intellectualism and empiricism, such transformation is neither the result of some form of explicit thought nor the result of mechanical responses to stimuli. It is effected through, as he puts it, "the motor grasping of a motor significance." 29

We believe that it makes sense to think of certain types of shared cultural patterns of understanding and action as habitually incorporated in this fashion. Individuals appropriate many such patterns practically, and through continual practice they make their way deeper into the primordial and pre-reflective bodily layer of our being. The patterns eventually congeal as

${ }^{27}$ Cf. Leder, The Absent Body, p. 179 n. 67: "the incorporation of a tool always involves the concurrent mastery of the skill whereby it is employed".

28 As Maria Talero writes: “The bodily 'past' of habit normally remains hidden within the very fabric of the present, 'submerged' in it as its beating heart. It is by withdrawing themselves from the forefront of my awareness that habits give efficacy and sense to my actions and sustain a world of meaningful projects around me.” See Talero, "Merleau-Ponty and the Bodily Subject of Learning," International Philosophical Quarterly 46(2) (2006): 191-203, at p. 195.

${ }^{29}$ Merleau-Ponty, Phenomenology of Perception, p. 165. 
more or less fixed socially shared habits. They become the things "one just says", what "we simply do" and not least what we instinctively feel that "we should do". We thus, to borrow a couple of useful terms from Sara Ahmed, "orientate" ourselves along and reproduce certain collective "lines" that direct our perception, thought and agency in some ways rather than others, and towards some things rather than others. ${ }^{30}$

This is a plausible phenomenological account of how, for instance, women (through the repeated "plucking, painting, toning, tanning, starving" that we have seen Benson describe) comply with society's standards of female looks and at the same time come to identify with these standards and take them up into their world-view. It also brings out how the different feminine modalities of bodily comportment and movement that Young details are not just the result of objectification of women and the imposition of sexist standards on them (from without, so to speak), but also shaped and upheld (from within) by their own active appropriation. ${ }^{31}$

The idea that many pervasive cultural norms are habitually incorporated helps explicate a feature of such norms that feminists have sometimes pointed towards: their elusiveness. These norms are primarily at work in the pre-thematic, taken-for-granted domain of bodily existence and co-existence, in the domain of the habitual. As incorporated, they belong to that from which we make sense of the world, act and interact, and thus rarely present themselves as that to which we direct our attention. Thus, these norms, and the preferences that they engender, tend to evade the sort of self-reflective scrutiny of our motivational structure that is crucial to autonomous agency. They are, in a sense, often too intimate to admit the distancing required for that kind of scrutiny.

\footnotetext{
${ }^{30}$ Sara Ahmed, Queer Phenomenology: Orientations, Objects, Others (Durham: Duke University Press, 2006).

${ }^{31}$ Although Young identifies the objectifying gaze of patriarchal society as the main source of these modalities, she also notes that "the woman often actively takes up her body as a mere thing". See Young, "Throwing Like a Girl," p. 155.
} 
Note how this account differs from many other accounts of how autonomy is set aside. On a procedural model, failures of autonomy are often construed as failures to bring higher order reflection to bear on explicit motives. Consider as an example the unwilling addict, who upon reflection does not really want to desire the drug, but cannot resist giving in to that desire when abstinence kicks in. ${ }^{32}$ Or consider the robbery scenario, where the victim's desire to give up her money is not endorsed as a motive she really wants but is forcibly induced by the sight of the robber's gun. ${ }^{33}$ The suggestion that many cultural norms are habitually incorporated entails that they generally do not have the degree of explicitness that the motives in these cases are assumed to have. Because of their pre-reflective and practical nature, one's corporeal habits are not accessible to straightforward voluntary introspection. They elude one no matter how hard one tries to think about them. ${ }^{34}$ On our account, the reason that actions influenced by pervasive norms are often non-autonomous is not that these norms have not been appropriately reflected upon and endorsed, but that they often do not present themselves as objects of reflection in the first place. The problem here is not that norms render reflection impotent, as addiction and

32 This well-known example is borrowed from Frankfurt, "Freedom of the Will and the Concept of a Person," pp. 12-14, but slightly modified. Frankfurt's story does not tell whether the desire for the drug or the desire not to desire the drug wins out in the end; the point is that the presence of second type of desire is what makes the addict a person rather than a wanton.

${ }^{33}$ Dworkin discusses the robbery case along these lines (briefly) in The Theory and Practice of Autonomy, p. 155, and (at more length) in "Acting Freely," Noûs 4(4) (1970): 367-83, at pp. 381-83. The case is, however, something of a standard example in the philosophical discussion of autonomy, coercion, and related concepts, and other authors have analysed it differently and for different purposes. See e.g. Lennart Nordenfelt, Rationality and Compulsion: Applying Action Theory to Psychiatry (Oxford: Oxford University Press, 2007), pp. 148-49.

${ }^{34}$ For an expansion on this point, see Talero, "Merleau-Ponty and the Bodily Subject of Learning," pp. 196-99. 
threats often do, but that they do not invite it. They tend to manifest themselves not by way of reflective consciousness but rather in what one unreflectively and habitually says, does, feels and sees.

This also means that our account is different from accounts of failure of "normative competence" put forward by proponents of substantive models of autonomy - models that do not regard a certain form of reflection on motives or preferences as sufficient for autonomy but also require that their content be of a certain type. It suggests that arguments to the effect that agents who act on preferences that reflect internalised, oppressive norms fail to act autonomously because they fail to recognise these norms as morally false $\mathrm{s}^{35}$ do not present the whole story of internalisation. We have argued that the problem, if internalisation is thorough enough, may not be so much that agents do not see that the norms that motivate their actions are false, but rather that they do not see them at all. And this may be the case regardless of the normative content of the norms in question. ${ }^{36}$

\section{Ex-corporation and critical distance to norms}

We have suggested that it is difficult to relate autonomously to many cultural norms because these norms are lived habitually and pre-reflectively rather than present as objects of reflection. Note that it is not suggested that we always fail to be autonomous with respect to the influence of such norms. Rather, it is a question of the shape that the failure of autonomy may take when we do

\footnotetext{
${ }^{35}$ Benson, "Autonomy and Oppressive Socialization"; Stoljar, "Autonomy and the Feminist Intuition".

${ }^{36}$ Hence on our account the failure to be autonomous with respect to oppressive or otherwise objectionable cultural norms is no different than the failure to be autonomous with respect to morally neutral or approvable norms. (No different, that is, in the sense that it is explained along the same lines - it is of course more troubling morally speaking.) For proponents of "normative competence" accounts the two kinds of failure obviously have to be construed differently - only the first involves a failure of correct normative understanding.
} 
fail. We certainly can reflect on and resist the influence of deeply entrenched cultural norms, and it seems clear that we sometimes do. But how are the opportunities for such reflection and resistance to be understood, more precisely, given the difficulties that have already been identified?

Generally speaking, the incorporated and habitual, while for the most part silently at work, is never wholly unintelligible and uncontrollable. As pointed out above, we retain a certain marginal awareness of incorporated objects, an awareness that may sometimes give way to explicit attention, as when the blind man in the above example shifts his focus from the world perceived at the end of his stick to the stick itself. And surely, not even the most deeply entrenched bodily habits are immutable. The "force" of habit is not strictly speaking a force, in the sense that the term has in physics, but a more flexible kind of power - albeit one that is neither introspectively accessible, nor to be undone by some sheer act of will.

We suggest that while the phenomenon of incorporation explains how certain cultural norms conceal themselves and thus elude autonomous reflection, the emergence of such norms as objects of scrutiny and control can be understood in terms of the converse phenomenon: ex-corporation. (Note that here as before the norms under discussion are those that require different kinds of bodily expression.) We have seen that incorporation is usually a gradual process, and that one often initiates it voluntarily only later to be carried along by it unreflectively, such as when one learns a skill like dancing. Ex-corporation, by contrast, typically occurs suddenly and unexpectedly.

Martin Heidegger's well-known example of the hammer in Being and Time is a useful illustration. The example is introduced in order to explain how human existence - understood as being-in-the-world - does not in the first place encounter the things that surround it as isolated objects with distinct and well-defined properties. Heidegger, like Merleau-Ponty, regards such a distanced and theoretical relation to them as secondary to, and founded upon, a more intimate practical familiarity. In his terminology, things primarily exist for us as equipment or tools (Zeuge). 
They have an "in-order-to..." structure, which means that what they are is a matter of what they allow us to do, and that they point us beyond themselves to the context of meaning in which they are situated. ${ }^{37}$ The hammer is essentially a tool for hammering, and as such it refers to what that activity aims at, say the construction of a house. Further, it refers to the people for whom the house is intended, as well as to the materials, instruments and other activities drawn together in the construction process. Ordinarily, we rely on such systems of reference confidently but unthematically. This is especially true as regards the tools that we are most immediately engaged with. The carpenter uses the hammer skilfully, but does not pay explicit attention to it, caught up as he is by the activity whereby it is used. But when it suddenly breaks and the hammering is brought to a stop, the hammer immediately emerges from its hiddenness and stands out as a conspicuous obstacle, obstructing the activity that it usually permits. And the network of references where it is situated is thereby revealed too. ${ }^{38}$

Drawing on the terminology we used earlier, we may say that when in normal use, the hammer is incorporated: it is a transparent medium that allows the body to extend itself into and actively engage the world in certain ways. ${ }^{39}$ But when the hammer malfunctions it ceases to be such a taken-for-granted extension of the carpenter's body schema. It no longer aligns itself with his hand and arm to form that from which he can proceed with his task, but is itself obtrusively brought forth as something to which he must attend. The hammer becomes ex-

\footnotetext{
${ }^{37}$ Martin Heidegger, Being and Time, trans. Joan Stambaugh (Albany, N.Y.: State University of New York Press, 1996), $\$ 15$.

38 Ibid., \16.

39 This way of elaborating the example departs from Heidegger himself, insofar as he generally has very little to say about the body, and does not mention it at all in the passages referred to here. However, several authors have explicitly linked his tool analysis to an analysis of the lived body. See, for instance, Leder, The Absent Body and Ahmed, Queer Phenomenology.
} 
corporated. It suddenly does not fit the carpenter's hand in the way required to efficiently drive nails; its malfunctioning is thus a "failure...to extend a body.",40

Like instruments, the bodily habits on which we for the most part tacitly draw to make sense of and act towards the things and people around us can be rendered explicit through ex-corporation. Consider, for instance, the incapacitation due to an injury and the ensuing process of rehabilitation. After the injury one may find oneself able to perform only with great difficulty everyday actions that one earlier just went on and did, such as walking or signing one's name. One is still capable of the bodily movements that these actions involve, but while they previously appeared to simply happen, remaining themselves transparent while allowing one to focus on other things, they now require explicit attention and effort. And rehabilitation often works through deliberately seizing and prolonging this initially involuntary ex-corporation. It aims, of course, at bringing back into habitual, pre-reflective bodily praxis movements that have been severed from it. But this often requires precisely the kind of thematisation that one seeks to make unnecessary - one repeatedly revisits movements that one before the injury relied on to simply happen, but that one now is required to struggle deliberately with, in order to be able once again to rely habitually and unthinkingly on them.

We may also become explicitly aware of our habits via the gaze of others. The things that we do quite spontaneously and unreflectively may strike other people as funny, inappropriate, disgusting and so on. We may sense their amusement, disapproval or disgust, and become aware of our behaviour that way. ${ }^{41}$ Individual habitual quirks are easily thematised in this manner because they are often conspicuous to others even in rather ordinary situations. But

\footnotetext{
40 Ahmed, Queer Phenomenology, pp. 49, 50

${ }^{41}$ For Jean-Paul Sartre, such objectification through the gaze of the other is the primordial form of bodily thematisation. See Sartre, Being and Nothingness: An Essay on Phenomenological Ontology, trans. Hazel E. Barnes (London: Routledge, 1969). See also Simone de Beauvoir The Second Sex, trans. and ed. H.M. Parshley (London: Campbell, 1993).
} 
socially widely shared forms of habitual behaviour are much more resistant to this form of excorporation. This is because individual instances of such behaviour are precisely not conspicuous, but rather "in line" ${ }^{42}$ - what everybody else habitually does and expects one to do.

However, even widely shared and deeply ingrained social practices and norms are capable of emerging from the pre-reflective bodily existence where they ordinarily remain concealed, and calling for attention. Consider so-called cultural clashes. In many such cases, the habitual praxis on which one usually relies in one's interaction with others fails to allow one's body to extend itself smoothly into an unfamiliar social and cultural space. As a mundane example, suppose that where I come from, strangers to whom one is introduced by a common acquaintance are greeted with a handshake. If I travel to a country where the appropriate way to greet people is instead with a kiss on each cheek, and upon being presented to someone I extend my hand as I always do, my gesture, which in my usual circumstances passes by quite unnoticed, now unintentionally attracts everybody's attention. My body and the cultural habit that it incarnates stand out as odd, not only to the people around me but to myself as well.

Ex-corporation carries an important reflective and critical potential. We have seen that habitual forms of understanding and behaviour are often, because of their embodied and practical nature, not directly accessible to voluntary acts of consciousness. But when they unexpectedly fail to extend our bodies in the way they usually do - when they become excorporated - they suddenly reveal themselves. When they temporarily "malfunction", they lose their transparency, emerge out of their concealment within the pre-reflective from structure of embodied experience, and present themselves as objects to which we may adopt a certain distance. This provides us with the opportunity to reflect on them - and indeed feel ashamed of or proud of them, joke about them and so on - in ways that we earlier could not. We may then also begin to grasp their role in shaping our experience of and engagement with the world.

\footnotetext{
42 This term, too, is borrowed from Ahmed, Queer Phenomenology.
} 
This opportunity for reflection may not be so important with respect to practices like handshakes, but it is crucial when it comes to pervasive and oppressive cultural habits that feminists and other social critics are concerned about, for instance norms about feminine physical appearance. This is because a reflective awareness of cultural habits is a precondition for all forms of sustained evaluation and criticism of them. And only after they have emerged from their bodily taken-for-grantedness can we begin to decide whether or not we want them to shape particular actions of ours. So ex-corporation opens up a space for autonomy with respect to pervasive cultural norms. This is of course not sufficient to guarantee that autonomy actually ensues, but at least it brings within the scope of autonomous scrutiny ways of seeing and doing things that were previously not up for such scrutiny. Ex-corporation may also inaugurate a process of changing pervasive norms, on an individual and societal level. ${ }^{43}$

Consider an imagined but plausible analogy to the failed handshake example, but more apt from a feminist viewpoint. A woman who quite habitually and unreflectively complies with her society's standards for feminine physical appearance and bodily comportment travels abroad. She is suddenly made painfully aware of these standards when she realises that meeting them does not allow her to smoothly fit in with the foreign culture's social life, but quite to the contrary causes ridicule. This awkward experience may make her own culture's standards available for her as objects of reflection and repudiation in a way that they earlier were not. She may even become a vocal public critic of them. A crucial thing to note about this reflection and critique is its being contingent on the unexpected. Many of our culturally shared habits are not fully grasped just by thinking about them. Rather, our thinking often needs to be incited by unforeseen and involuntary events; it requires an unintended breakdown in ordinary, habitual understanding.

\footnotetext{
${ }^{43}$ Obviously, because of the practical and habitual nature of these norms, a mere intellectual rejection of them, and an ability to avoid their motivating particular actions, is not sufficient for changing them. Change would also have to include a form of reverse habituation, i.e. a gradual practical transformation.
} 
It is important to note that the qualification made earlier applies here too. Just like the phenomenon of incorporation was invoked to describe the concealment of norms that are to some degree corporeally enacted, the phenomenon of ex-corporation is primarily meant to elucidate how individuals may begin to relate autonomously to and strive to change norms of this particular kind. The story is likely to be different when it comes to culturally shared forms of understanding that are more like the property right norm discussed earlier. Norms of that kind are at work not so much in individual bodily praxis as in political and institutional contexts. Calling attention to and challenging them does not require an unintended breakdown in embodied understanding because they are already in a largely reflective and discursive realm. They may sometimes be as elusive as bodily habits, but their elusiveness is of another kind and is hence to be undone in other ways. They are likely to be more accessible for straightforward social critique and consciousness-raising.

This is not to deny a role for such deliberate efforts with respect to questioning and changing corporeal habits too. Rather, they tend to be the most effective when they successfully incorporate an element of genuine surprise, when they jolt us out of our taken-for-granted bodily ways of making sense of things. Interestingly, feminist and other critical forms of art and literature often appear to work in precisely this way.

\section{The two-sex model and parents' experiences of children born with ambiguous sex}

After this theoretical analysis of how certain cultural norms shape individuals' experiences and actions we now turn back to the case with which this paper started: the birth of a child with ambiguous sex. We will apply our analysis to the findings from empirical studies of parental experiences of receiving such a child, and of the ensuing process of sex assignment and genital surgery. Our concern in this final section of the paper is not so much with autonomous reflection and agency as with contributing to an understanding of these experiences. 
The birth of a child with ambiguous sex brings to the fore norms that run particularly deep in our culture - norms, moreover, to which our previous analysis applies well. The two-sex model, ${ }^{44}$ i.e. the idea that humanity is and must be made up of two sexes that are defined in terms of their opposition to each other, an opposition that is to a large extent determined by genital appearance, is arguably one of the most basic principles around which we structure the social world. It shapes basic societal institutions - think, for instance, of marriage and the family - as well as intellectual and discursive practices such as scientific investigation. ${ }^{45}$ But it also pervasively influences everyday social interaction and the complex forms of bodily movement and expression involved therein. The model is not only a conceptual structure, but also (and arguably primarily) something that we live as bodily, gendered beings. It is plausibly thought of as incorporated into our taken-for-granted pre-reflective bodily existence. The norms concerning sexual difference and genital appearance that constitute the model are integrated into the from structure of the lived body: we habitually perceive and act upon the world from or through them. Thus they are not themselves the focus of attention. In our everyday lives we rely on them constantly but quite tacitly to orientate ourselves in social space.

We soon turn to how this embodied understanding shapes how parents experience the birth of an ambiguously sexed child and the medical process that follows. But first a few words to put that situation in context. There are many different conditions that may cause a child to be born with chromosomes, hormone levels and external and internal genitals that cannot all be classified as belonging to the same sex. As a result of such a condition a newborn child may have female genital appearance but male chromosomes, undeveloped testicles and no uterus or

\footnotetext{
${ }^{44}$ The term is borrowed from Thomas Laqueur, Making Sex: Body and Gender from the Greeks to Frend (Cambridge, Mass.: Harvard University Press, 1990).

${ }^{45}$ For different versions of the claim that gender norms shape science, see the collection of essays in Evelyn Fox Keller and Helen E. Longino (eds.), Feminism and Science (Oxford: Oxford University Press, 1996).
} 
ovaries, or female chromosomes and either male external genitalia or so-called virilised female genitalia. Medical professionals in paediatric endocrinology have recently agreed internationally to use the term "disorders of sex development" (or DSD) as an umbrella term for these conditions. ${ }^{46}$ We will, however, use the terms "intersex" and "ambiguous sex", interchangeably, since these are the terms that patients' organisations have so far preferred.

When a child is born with ambiguous sex, medical professionals consider a thorough assessment and decision regarding the child's sex to be required as soon as possible. ${ }^{47}$ In most cases, however, the reason for the urgency is not medical but social. Parents find it very difficult not to be able to tell relatives and friends what the child's sex is. ${ }^{48}$ Also, medical professionals stress that the child needs to be assigned a sex so that the parents can bring it up as what is considered a normal boy or girl. This is also the reason why it is often deemed necessary or desirable to have the child undergo surgery that makes its genitalia look normal for the assigned sex. ${ }^{49}$ There is international agreement among paediatric endocrinologists that genital surgery, if performed, should generally be done in an early phase of the child's life. ${ }^{50}$ In many cases one operation is later followed by more operations.

46 Peter A. Lee, Christopher P. Houk., S. Faisal Ahmed. \& Ieuan A. Hughes, "Consensus Statement on Management of Intersex Disorders," Pediatrics 118 (2006): e488-e500. DSD is also used as a medical term for hypospadic boys, i.e. boys that do not have the urethral opening located on the tip of the penis.

${ }^{47}$ Ibid., p. e491.

${ }^{48}$ Sanders et al., "Parents' narratives about their experiences of their child's reconstructive genital surgeries for ambiguous genitalia," p. 3191.

${ }^{49}$ C. Nihoul-Fékété, ”Does Surgical Genitoplasty Affect Gender Identity in the Intersex Infant?” Hormone Research 64 (suppl. 2) (2005): 23-26.

${ }^{50}$ Lee et al., "Consensus Statement on Management of Intersex Disorders," p. e492. 
The surgery has been the target of criticism from patients' organisations and scholars. Some patients who have had operations assert that the operations have created precisely the sense of abnormality that they were designed to avoid. ${ }^{51}$ They also describe how their sexual function and pleasure have been impaired ${ }^{52}$ Criticism has also been directed at the parents' proxy consent. It has been suggested that surgery should be postponed until the child can participate in the decision. ${ }^{53}$

Parents' stories about the events surrounding the birth of an intersex child are marked by deep confusion, frustration and disbelief. ${ }^{54}$ Many parents describe the realisation that doctors do not know the sex of their newborn child as bewildering. As one father put it: "We were just confused obviously, what don't, why don't they know what he is?" 55 Many also report that the medical staff declared a specific sex of their child at birth but changed opinion some hours or days later, or that different doctors had different opinions regarding their child's sex. During this first state of uncertainty, parents learn that genitals may be ambiguous, that sex does not primarily have to do with genitals and that their child is a complex and difficult case.

${ }^{51}$ Sharon E. Preves, "Negotiating the Constraints of Gender Binarism: Intersexuals' Challenge to Gender Categorization," Current Sociology 48 (2000): 27-50, at p. 32. See also Alice Domurat Dreger, Hermaphrodites and the Medical Invention of Sex (London: Harvard University Press, 1998).

52 Suzanne J. Kessler, Lessons from the Intersexed (New Brunswick: Rutgers University Press, 1998), pp. 5761.

${ }^{53}$ Kenneth Kipnis \& Milton Diamond, "Pediatric ethics and the surgical assesment of sex," Journal of Clinical Ethics 9(4) (1998): 398-410. See also Dreger, Hermaphrodites and the Medical Invention of Sex.

${ }^{54}$ Unless otherwise stated, the descriptions of parental experiences in what follows draw on the systematisation and analysis of research on such experiences in Kristin Zeiler and Anette Wickström, "Why do 'we' perform surgery on newborn intersexed children? The phenomenology of the parental experience of having a child with intersexed anatomies," (forthcoming in Feminist Theory).

55 Gough et al., "They did not have a word," p. 499. 
Soon after the child's birth, medical professionals start the process of biologically defining the child's sex, through a search for different marks on or in the body. This sex assignment process includes chromosome tests, ultra-sound, hormone treatment and measurement of the response to the hormones. Many parents seem to experience themselves as being in a "liminal state of waiting" for a result at this stage of the process. ${ }^{56}$ In the absence of decisive medical information they anxiously search for informal ways to categorise their child as belonging to one sex or the other..$^{57}$ To some, it is as if they have not had a child yet, because a child is supposed to be either a boy or a girl. One mother explained that she "just felt numb you know and, I didn't, I couldn't register with, with her anyway, 'cos I didn't even know what she was!" 58

The dual phenomena of incorporation and ex-corporation described earlier in this paper help to elucidate these experiences. We have suggested that the two-sex model is a fundamental structuring principle for social life and that much of its influence is exerted on the habitual and pre-reflective bodily level of human existence and coexistence; i.e. that it is incorporated. When parents are faced with an infant whose sex cannot be immediately determined, this basic pattern of understanding is all of a sudden disrupted. They cannot rely on it to make sense of this new situation that they find themselves in, and the sense of orientation that it provided is lost. One important reason why this is so baffling and frustrating is precisely that their dependence on the model has been to a great extent practical and embodied, rather than intellectual, and because, for that very reason, it has tended to elude their attention. Now the

\footnotetext{
${ }^{56}$ Zeiler and Wickström, "Why do 'we' perform surgery on newborn intersexed children?"

${ }^{57}$ Gough et al., "They did not have a word," pp. 501-503.

${ }^{58}$ Ibid., p. 499. For other parental stories about parents' difficulty in relating to intersexed children, see Sanders et al., "Parents' narratives about their experiences of their child's reconstructive genital surgeries for ambiguous genitalia," pp. 3190-91.
} 
model suddenly emerges as defective; it becomes ex-corporated. It is useful here to recall some of the features of that phenomenon highlighted earlier.

First, the ex-corporation of the two-sex model involves its failure to give the parents full access to a social world, just as the malfunctioning of the hammer in Heidegger's example involves its failure to give the carpenter access to the network of meaning that uninhibited hammering normally tacitly refers him to. Their pre-reflective, habitual way of understanding sexual difference and genital appearance suddenly does not "fit the world". It does not allow the parents to seamlessly extend their bodies towards their newborn child in the way they usually extend them towards other cohabitants of that world. This helps explain some parents' reported difficulty in relating to the child as theirs. Second, this breakdown in ordinary understanding renders that understanding itself explicit. Deeply entrenched habits that have previously eluded parents' attention, precisely because they have been that from which they make sense of the world, now suddenly emerge as something problematic that must be attended to. Hence it is evident from parents' stories that their child's birth has made them doubt what they had previously taken for granted about sexual difference and genital appearance. Third, this new and heightened awareness is not gained voluntarily - parents do not suddenly decide to think about what was earlier only practically and habitually grasped. It is instead called forth by a highly unexpected, even shocking, event.

This breakdown and simultaneous rendering explicit of the parents' embodied understanding of sex and the importance of genitals, provoked by the child's atypical body, presents a double-faced situation. This is evidenced by their responses to that situation, which tend in two opposite directions. On the one hand, doubts may give way to more sustained questioning of the two-sex model. The sudden visibility of previously invisible, self-evident understanding may be seized as an opportunity to reflect on, and even modify that understanding. The lack of fit between parents' embodied understanding of sex and the world 
that they encounter, and more specifically their newborn child, is on this stance managed through a modification of the former.

Hence some parents declare that as a result of their child's birth and the medical process of sex assignment, they now see sex as much more "fluid" than before. Some say they start to accept more diversity in being human, and that a richer variation in gender roles seems acceptable to them. Some parents also find it strange that external genitals are given such importance by the medical staff. When they learnt about their child's ambiguous sex, they started to question the two-sex model, including the idea that external genitals are crucial with regard to someone's sex. This happened when one father, whose child had XX chromosomes, was told by the medical staff that the child "would make a very nice boy" because of its genitals. "In my mind," he commented, the chromosomes made the child a girl "irrespective of the appearance of the genitalia". ${ }^{59}$

On the other hand, however, the opposite tendency is the dominant one. When the sex assignment process is completed (which may take up to six months, even though it should ideally not take more than a few weeks) and the child is assigned female or male sex, the parents are typically greatly relieved. While they have previously been told not to give a name to their baby, to speak of it as a boy or girl or refer to it as "he" or "she", they are now asked to do so. Some parents say that their child appears to them as a human being for the first time at this stage. It is also now that parents are asked whether they want to have the child undergo surgery in order to make its genitals look more typical for the assigned sex. They usually welcome this offer, despite the fact that such surgery is, in most cases, not medically necessary. ${ }^{60}$ Indeed, medical

\footnotetext{
${ }^{59}$ Gough et al., "They did not have a word," p. 502.

${ }^{60}$ Kessler, Lessons from the Intersexed, p. 32.
} 
professionals emphasise that parents request early surgical intervention. ${ }^{61}$ So while the birth of an intersex child temporarily sheds doubt on the two-sex model, the model is quickly vindicated. The failure of "fit" between the child and the parents' embodied understanding of the two sexes as starkly opposed and of genital appearance as decisive for one's belonging to one them is not taken as an occasion to seriously question or modify that understanding. Rather, the child is modified instead.

In order to understand this response it is important to bear in mind the medical context in which the parents find themselves. It has been suggested that both medical knowledge and clinical practice are informed by and reproduce deeply rooted norms about sexual difference. ${ }^{62}$ And the idea that genital appearance is crucial to children's (and parents') wellbeing has played an important role in the medical management of intersex conditions. ${ }^{63}$ Also, parents of newborn children with ambiguous sex are in an extraordinarily vulnerable situation, where their dependence on the medical staff and the knowledge that they represent is great. ${ }^{64}$ It is also important to remember that the parents may not have known before their child was born that not all children can be easily categorised as girls or boys immediately after birth.

Making sense of parents' urge for sex assignment and often for surgery also requires bearing in mind some of the features of embodied cultural norms that our analysis has

${ }^{61}$ Ethelwyn Rebelo, Christopher P. Szabo \& Graeme Pitcher, "Gender assignment surgery on children with disorders of sex development: A case report and discussion from South Africa," Journal of Child Health Care 12(1) (2008): 49-59, at p. 52.

62 For an argument along these lines specifically focusing on the theory and practice surrounding the management of intersex conditions, see Kessler, Lessons from the Intersexed.

${ }^{63}$ For a recent defence of that idea, see Nihoul-Fékété, ’Does Surgical Genitoplasty Affect Gender Identity in the Intersex Infant?"

${ }^{64}$ Sanders et al., 'Parents' narratives about their experiences of their child's reconstructive genital surgeries for ambiguous genitalia," pp. 3191-92 and Ellen K. Feder, "In Their Best Interests". 
brought out. Even if the birth of an intersex child makes the two-sex model emerge as defective and allows parents to question it, they rely on that model so heavily and in so many areas of their lives (as indeed all of us do) that it is exceedingly difficult to maintain a questioning stance towards it. The model is presupposed in most forms of everyday social interaction, as the means of access to a shared world. If that interaction is to function smoothly, through the model, the model cannot itself remain thematised as problematic or defective, but must disappear into prereflective bodily praxis. Thus, encounters with other people constantly work towards a reincorporation of the model.

The sudden ex-corporation of the previously taken-for-granted understanding of sex and genitals when an intersex child is born not only involves the becoming apparent of that understanding itself. Parents may also get a sense of its indeed pervasive role in social life. ${ }^{65}$ It may suddenly strike them just how heavily the two-sex model would weigh on someone that cannot be categorised according to its standards. Hence the child's birth typically gives rise to worries about what other people will think and how they will respond to the child in a host of different situations. ${ }^{66}$ Such worries may make questioning of the two-sex model on the parents' own part appear futile, as the widely shared character of that model suddenly becomes apparent. And they quite understandably reinforce the sense that quick sex assignment and surgery is necessary for the child's well-being.

In the light of all this, it is small wonder that parents experience the birth of a child with ambiguous sex as deeply confusing and frustrating, and that they often so eagerly embrace

${ }^{65}$ Here, again, is an analogy with Heidegger's hammer. As we have seen, the malfunctioning hammer not only reveals itself, but also calls attention, in different ways, to other things in the practical context where it is used, things that were previously simply taken for granted.

${ }^{66}$ Parents are for instance concerned that the child will be teased and socially excluded. See Gough et al, “They did not have a word", p. 503; and Sanders et al., "Parents' narratives about their experiences of their child's reconstructive genital surgeries for ambiguous genitalia,” p. 3191. 
the offer of genital surgery for the child. The two-sex model, we have argued, exerts a considerable but silent power on such experiences and decisions. An awareness of that power is important for clinical practice concerning intersex children, not least for the development of adequate procedures for parents' informed consent to the different interventions involved. Such awareness is also important for critics of that practice. If we are right about how parental choices are shaped by pervasive but largely taken-for-granted cultural norms about sexual difference and genital appearance, then the scope for holding individual parents responsible when their choices end up harming children is limited. Criticism is then more appropriately to be levelled at these norms and the ways in which they are taken up into and amplified by medical practice. To end on an optimistic note, our analysis should give critics of the medical management of intersex conditions some reassurance, because it suggests that even very elusive and deeply entrenched norms can be questioned and changed. 